\title{
Ruin Probabilities for a Sparre Andersen Model with Investments
}

\author{
Ernst Eberlein \\ University of Freiburg, Department of Mathematical Stochastics, Ernst-Zermelo-Str. 1, 79104 \\ Freiburg, Germany \\ Yuri Kabanov \\ Lomonosov Moscow State University, Russia, and Laboratoire de Mathématiques, Université de \\ Franche-Comté, 16 Route de Gray, 25030 Besançon, cedex, France \\ Thorsten Schmidt \\ University of Freiburg, Department of Mathematical Stochastics, Ernst-Zermelo-Str. 1, 79104 \\ Freiburg, Germany
}

\begin{abstract}
We study a Sparre Andersen model in which the business activity of the company is described by a compound renewal process with drift assuming that the capital reserves are invested in a risky asset. The price of the latter is assumed to evolve according to a geometric Lévy process. We prove that the asymptotic behavior of the ruin probability depends to a large extent only on the properties of the price process.
\end{abstract}

Keywords: Ruin probabilities, Sparre Andersen model, Actuarial models with investments, Renewal processes, Distributional equations

MSC 60G51, 60G70, 91G05

*This work was done during the stay of the second author at the Freiburg Institute for Advanced Studies (FRIAS), Germany; it was supported by the Russian Science Foundation grant 20-68-47030. The third author gratefully acknowledges the support by the DFG with grant SCHM 2160/15-1.

Email addresses: eberleinestochastik .uni-freiburg.de (Ernst Eberlein), ykabanov@univ-fcomte.fr(Yuri Kabanov),

thorsten.schmidtestochastik.uni-freiburg.de (Thorsten Schmidt) 


\section{Introduction}

In the classical collective risk theory the Sparre Andersen model (called also renewal risk model) is a generalisation of the Lundberg-Cramér model in which it is assumed that the claims count is a renewal process rather than a Poisson process, i.e. the times between consecutive claims are independent random variables which are not necessarily exponentially distributed, see, e.g., Grandell's book [4]. The word "classical" denotes the situation in which the reserves of the insurance company are kept in a bank account which does not earn any interest. In the modern literature more realistic models are considered, namely those in which the capital is invested, in full or partially, in a risky financial asset. It was shown in [3] that the financial risk leads to a dramatic change in the behavior of the ruin probability $\Psi(u)$ as a function of the initial capital $u$ : instead of its comfortable exponential decrease with the growth of the available capital, in case of the investment of the reserves in the stock market, the ruin probability $\Psi(u)$ decreases as a power function. Moreover, the ruin is imminent, $\Psi \equiv 1$, if the volatility of the stock market is too high. Because of the practical importance of this phenomenon, actuarial models with investments attracted a lot of attention. Most of the papers deal with asymptotic studies in settings stemming from the LundbergCramér model and can be included in the beautiful mathematical framework of the generalised Ornstein-Uhlenbeck process suggested and studied by Paulsen, see [8] - [11]. Paulsen used the powerful method of distributional equations. A detailed analysis of the available results is beyond the scope of the present note and we refer the reader to the recent paper [6].

In contrast to the Lundberg-Cramér line of research there are very few results on ruin probabilities for Sparre Andersen type models with investments. The paper by Albrecher, Constantinescu, and Thomann [1] is one of the rare studies to the latter, see also references therein. Their approach is heavily based on the techniques of continuous time Markov processes,

In the present note we consider a Sparre Andersen type non-life insurance model with investment in a risky asset. The price of this asset evolves according to an exponential Lévy process, while the business activity of the company is described by a compound renewal process with positive drift and negative jumps. The asymptotic result which is achieved is basically of the same form as the main result of [6]. It requires only very weak assumptions on the interarrival times, like existence of the exponential moments, which are fulfilled in the case of the expo-

nential distribution. As in [6], the main assumption is that the cumulant generating function $H: \gamma \mapsto \ln \mathbf{E} e^{-\gamma V_{1}}$ of the $\log$ price process $V$ has a strictly positive root 
$\beta$ which does not lie on the boundary of the effective domain of $H$. Assuming that the claims are random variables, whose power of order $\beta$ is integrable, the ruin probability decays with the rate $u^{-\beta}$ (see Theorem 2.3).

As in [6], our proofs are based on recent progress in the theory of distributional equations.

\section{The model}

Let $\left(\Omega, \mathcal{F}, \mathbf{F}=\left(\mathcal{F}_{t}\right)_{t \geq 0}, \mathbf{P}\right)$ be a stochastic basis on which there is given a non-deterministic Lévy process $R=\left(R_{t}\right)_{t \geq 0}$ with $\Delta R>-1$ and an independent compound renewal process $P=\left(P_{t}\right)_{t \geq 0}$ with drift $c>0$ and negative jumps. We denote by $T_{n}, n \geq 1$, the successive jump instants of the latter process and put $F(t):=\mathbf{P}\left(T_{1} \leq t\right)$. We associate with $R$ the stochastic exponential $\mathcal{E}(R)$ which is, due to the assumption on the jumps, a strictly positive process.

We will study the process $X=X^{u}, u>0$, which is defined as the solution of the non-homogeneous linear stochastic equation

$$
X_{t}=u+\int_{0}^{t} X_{s-} d R_{s}+P_{t} .
$$

The process $X$ can be written in the "dot" notation of stochastic calculus for as $X=u+X_{-} \cdot R+P$ or, in its "differential" form, as $d X_{t}=X_{t-} d R_{t}+d P_{t}$, $X_{0}=u$. The compound renewal process $P$ is usually represented in the form

$$
P_{t}=c t+\sum_{i=1}^{N_{t}} \xi_{i},
$$

where $N$ is a counting renewal process with interarrival times $T_{i}-T_{i-1}, i \geq 1$, which form an i.i.d. sequence, independent of the i.i.d. sequence $\xi_{i}=\Delta P_{T_{i}}$, $i \geq 1$. The case considered here $\left(c>0\right.$ and $\left.\xi_{i}<0\right)$ corresponds to the non-life insurance situation. For $R=0$ we have the classical Sparre Andersen model (which we already excluded by the assumptions made).

In the actuarial context $X=X^{u}$ represents the dynamics of the reserves of an insurance company that invests the total of these reserves in a stock with the price process $S=\mathcal{E}(R)$ which has unit as its initial value. Having in mind that $d R=d S / S_{-}$, it is natural to call $R$ relative price process, while $V:=\ln \mathcal{E}(R)$ is usually referred to as the log price process. Thus the price process $S$ which we introduced here as a stochastic exponential can alternatively be expressed as an ordinary exponential $S=\exp (V)$. For the interplay between stochastic and 
ordinary exponentials see Theorem 3.49 in [2]. Note that $V$ is again a Lévy process. In general this process will have jumps of arbitrary size in both directions. Typical examples which are used in financial models are generalized hyperbolic processes and various subclasses such as hyperbolic, normal inverse Gaussian or variance gamma processes.

The processes $R$ and $P$ generate the filtration $\mathbf{F}^{R, P}=\left(\mathcal{F}_{t}^{R, P}\right)_{t>0}$. Define $\tau^{u}:=\inf \left\{t: X_{t}^{u} \leq 0\right\}$ (the instant of ruin) and $\Psi(u):=\mathbf{P}\left(\tau^{u}<\infty\right)$ (the ruin probability).

Let $\left(a, \sigma^{2}, \Pi\right)$ be the Lévy triplet of $R$ corresponding to the standard truncation function $h(x):=x I_{\{|x| \leq 1\}}$. Putting $\bar{h}(x):=x I_{\{|x|>1\}}$, we can write the canonical decomposition of $R$ in the form

$$
R_{t}=a t+\sigma W_{t}+h *(\mu-\nu)_{t}+\bar{h} * \mu_{t} .
$$

Here $W$ is a standard Wiener process, $\mu(d t, d x)$ is the random measure of jumps of $R$, i.e. the Poisson random measure with compensator $\nu(d t, d x)=d t \Pi(d x)$.

For notations and basic facts we recommend the books [5], Ch. 2, and [2], Chs. 2 and 4. As in [6] the symbols $\Pi(f)$ or $\Pi(f(x))$ stand for the integral of $f$ with respect to the Lévy measure $\Pi$. Recall that $\Pi\left(x^{2} \wedge 1\right)<\infty$.

Due to the independence of $R$ and $P$, these processes have no common jumps. The solution of the generalised stochastic exponential (1) can be represented (see [2], Proposition 3.48) as

$$
X_{t}^{u}:=\mathcal{E}_{t}(R)\left(u+\mathcal{E}_{-}^{-1}(R) \cdot P_{t}\right) .
$$

Since we assume that $\Delta R>-1$ and $R$ is nondeterministic, the Lévy measure $\Pi$ is concentrated on the interval $]-1, \infty\left[\right.$ and the Lévy characteristics $\sigma^{2}$ and $\Pi$ do not vanish simultaneously.

Recall that the stochastic exponential $\mathcal{E}_{t}(R)$ has explicit form

$$
\mathcal{E}_{t}(R)=e^{R_{t}-\frac{1}{2} \sigma^{2} t+\sum_{s \leq t}\left(\ln \left(1+\Delta R_{s}\right)-\Delta R_{s}\right)} .
$$

The $\log$ price $V=\ln \mathcal{E}(R)$ can then be expressed as

$$
V_{t}=a t-\frac{1}{2} \sigma^{2} t+\sigma W_{t}+h *(\mu-\nu)_{t}+(\ln (1+x)-h) * \mu_{t} .
$$

Its Lévy triplet is $\left(a_{V}, \sigma^{2}, \Pi_{V}\right)$ where

$$
a_{V}=a-\frac{\sigma^{2}}{2}+\Pi(h(\ln (1+x))-h)
$$


and $\Pi_{V}=\Pi \varphi^{-1}, \varphi: x \mapsto \ln (1+x)$.

The cumulant generating function $H: q \rightarrow \ln \mathbf{E} e^{-q V_{1}}$ of the random variable $V_{1}$ admits an explicit expression

$$
H(q):=-a_{V} q+\frac{\sigma^{2}}{2} q^{2}+\Pi\left(e^{-q \ln (1+x)}-1+q h(\ln (1+x))\right) .
$$

Its effective domain $\operatorname{dom} H:=\{q: H(q)<\infty\}$ is the set $\{J(q)<\infty\}$ where

$$
J(q):=\Pi\left(I_{\{|\ln (1+x)|>1\}} e^{-q \ln (1+x)}\right)=\Pi\left(I_{\{|\ln (1+x)|>1\}}(1+x)^{-q}\right) .
$$

Its interior is the open interval $(\underline{q}, \bar{q})$ with

$$
\underline{q}:=\inf \{q \leq 0: J(q)<\infty\}, \quad \bar{q}:=\sup \{q \geq 0: J(q)<\infty\} .
$$

Being a convex function, $H$ is continuous and admits finite right and left derivatives on $(\underline{q}, \bar{q})$. If $\bar{q}>0$, then the right derivative satisfies

$$
D^{+} H(0)=-a_{V}-\Pi(\bar{h}(\ln (1+x)))<\infty,
$$

though it may be equal to $-\infty$. We do not exclude this case.

In the formulation of the asymptotic results we shall always assume that $\bar{q}>0$ and that the equation $H(q)=0$ has a root $\beta \in] 0, \bar{q}[$. Since $H$ is not constant, such a root is unique. Clearly, it exists if and only if $D^{+} H(0)<0$ and $\lim \sup _{q \uparrow \bar{q}} H(q) / q>0$. In the case where $\underline{q}<0$ the condition $D^{-} H(0)>0$ is necessary to ensure that $H(q)<0$ for values $q<0$ which are sufficiently small in absolute value.

If $J(q)<\infty$, then the process $m=\left(m_{t}(q)\right)_{t \geq 0}$ with

$$
m_{t}(q):=e^{-q V_{t}-t H(q)}
$$

is a martingale and

$$
\mathbf{E} e^{-q V_{t}}=e^{t H(q)}
$$

Lemma 2.1. Suppose that $E e^{\varepsilon T_{1}}<\infty$ for some $\varepsilon>0$. Let $\left.\beta \in\right] 0, \bar{q}[$ be the root of the equation $H(q)=0$. If $q \in[\beta, \bar{q}[$ is such that $H(q) \leq \varepsilon / 2$, then

$$
\mathbf{E} \sup _{s \leq T_{1}} e^{-q V_{s}}<\infty .
$$


Proof. Let us take $r \in] 1, \bar{q} / q[$ sufficiently close to 1 to ensure the inequality $H(q r)-r H(q))<(1 / 2) \varepsilon$. Then

$$
\mathbf{E} m_{t}^{r}(q)=e^{t(H(q r)-r H(q))} \leq e^{(1 / 2) \varepsilon t} .
$$

Using Doob's inequality we get that

$$
\mathbf{E} \sup _{s \leq t} m_{s}(q) \leq \mathbf{E} \sup _{s \leq t} m_{s}^{r}(q) \leq C_{r} e^{t(H(q r)-r H(q))} \leq C_{r} e^{(1 / 2) \varepsilon t} .
$$

Note that

$$
e^{-q V_{s}}=m_{s}(q) e^{s H(q)} \leq e^{t H(q)} \sup _{s \leq t} m_{s}(q) \leq e^{(1 / 2) \varepsilon t} \sup _{s \leq t} m_{s}(q) .
$$

Therefore,

$$
\mathbf{E} \sup _{s \leq T_{1}} e^{-q V_{s}}=\int_{0}^{\infty} e^{(1 / 2) \varepsilon t} \mathbf{E} \sup _{s \leq t} m_{s}(q) F(d t) \leq C_{r} \mathbf{E} e^{\varepsilon T_{1}}<\infty .
$$

The lemma is proven.

Corollary 2.2. Let $Q:=e^{-V} \cdot P_{T_{1}}$. If the conditions of the above lemma are fulfilled and $\mathbf{E}\left|\xi_{1}\right|^{\beta}<\infty$, then $\mathbf{E}|Q|^{\beta}<\infty$.

Proof. Take $q \in] \beta, \bar{q}[$ such that $H(q)<\varepsilon / 2$. Using an obvious estimate and applying the Hölder inequality with $r:=q / \beta>1$ and $p:=r /(r-1)$, we get that

$$
\mathbf{E}\left(\int_{\left[0, T_{1}\right]} e^{-V_{t}} d t\right)^{\beta} \leq \mathbf{E} T_{1}^{\beta} \sup _{s \leq T_{1}} e^{-\beta V_{s}} \leq\left(\mathbf{E} T_{1}^{p \beta}\right)^{1 / p}\left(\mathbf{E} \sup _{s \leq T_{1}} e^{-q V_{s}}\right)^{1 / r}<\infty .
$$

If $\beta \leq 1$, the elementary inequality ||$a|+| b||^{\beta} \leq|a|^{\beta}+|b|^{\beta}$ implies that

$$
\mathbf{E}|Q|^{\beta} \leq c^{\beta} \mathbf{E}\left(\int_{\left[0, T_{1}\right]} e^{-V_{t}} d t\right)^{\beta}+\mathbf{E} e^{-\beta V_{T_{1}}} \mathbf{E}\left|\xi_{1}\right|^{\beta}<\infty .
$$

If $\beta>1$, we use the inequality ||$a|+| b||^{\beta} \leq 2^{\beta-1}\left(|a|^{\beta}+|b|^{\beta}\right)$ and arrive to the same conclusion.

Our main result is the following theorem. 
Theorem 2.3. Suppose that there is $\beta \in] 0, \bar{q}\left[\right.$ such that $H(\beta)=0, \mathbf{E}\left|\xi_{1}\right|^{\beta}<\infty$. and $\mathbf{E} e^{\varepsilon T_{1}}<\infty$ for some $\varepsilon>0$. If $\sigma \neq 0$ or the law of $\left|\xi_{1}\right|$ has unbounded support, then

$$
0<\liminf _{u \rightarrow \infty} u^{\beta} \Psi(u) \leq \limsup _{u \rightarrow \infty} u^{\beta} \Psi(u)<\infty .
$$

If $\sigma=0$ and the law of $\left|\xi_{1}\right|$ has bounded support, (11) also holds except for the case where $0<\Pi(|h|)<\infty$ and $\Pi(]-1,0[) \Pi(] 0, \infty[)=0$. In the latter case one needs the additional assumption that $\mathbf{P}\left(T_{1} \leq t\right)>0$ for any $t>0$.

Remark. In [7] it is shown that in the model where $S$ is a geometric Brownian motion, $\beta:=2 a / \sigma-1 \leq 0$, and $\mathbf{E} e^{\varepsilon T_{1}}<\infty$ for some $\varepsilon>0$, the ruin probability $\Psi \equiv 1$.

\section{Ruin problem: reduction to a discrete time case}

Since we consider the non-life insurance model, ruin may happen only at jump-times of $P$. This allows us to monitor the process $X^{u}$ only along the sequence $T_{n}$, i.e. to reduce the problem to a discrete time setting. For this purpose we introduce the discrete time processes $V_{T_{n}}$ and $Y_{n}:=-e^{-V_{-}} \cdot P_{T_{n}}, n=1,2, \ldots$ Then

$$
X_{T_{n}}^{u}=e^{V_{T_{n}}}\left(u-Y_{n}\right) .
$$

Note that $Y_{n}$ is a Markov chain and

$$
Y_{n}=-\sum_{k=1}^{n} e^{-V_{T_{k-1}}} \int_{] T_{k-1}, T_{k}\right]} e^{-\left(V_{s-}-V_{T_{k-1}}\right)} d P_{s}
$$

Define the stopping time $\theta^{u}:=\inf \left\{n \geq 1: Y_{n} \geq u\right\}$ with respect to the discrete time filtration $\mathbf{G}=\left(\mathcal{G}_{n}\right)_{n \geq 1}$ where $\mathcal{G}_{n}:=\mathcal{F}_{T_{n}}^{R, P}$. In the considered model ruin happens only at the jump-times of $P$. Hence, $\left\{\tau^{u}<\infty\right\}=\left\{\theta^{u}<\infty\right\}$ and $\Psi(u)=\mathbf{P}\left(\theta^{u}<\infty\right)$.

Lemma 3.1. If $Y_{n} \rightarrow Y_{\infty}$ almost surely as $n \rightarrow \infty$, where $Y_{\infty}$ is a finite random variable unbounded from above, then for all $u>0$

$$
\bar{G}(u) \leq \Psi(u) \leq \bar{G}(u) / \bar{G}(0),
$$

where $\bar{G}(u):=\mathbf{P}\left(Y_{\infty}>u\right)$. 
Proof. Let $\theta$ be an arbitrary stopping time with respect to the filtration G. As we assume that the finite limit $Y_{\infty}$ exists, the random variable

$$
Y_{\theta, \infty}:= \begin{cases}-\lim _{N \rightarrow \infty} \int_{]_{T_{\theta}}, T_{\theta+N}\right]} e^{-\left(V_{t-}-V_{T_{\theta}}\right)} d P_{t}, & \theta<\infty, \\ 0, & \theta=\infty,\end{cases}
$$

is well defined. On the set $\{\theta<\infty\}$

$$
Y_{\theta, \infty}=e^{V_{T_{\theta}}}\left(Y_{\infty}-Y_{\theta}\right)=X_{T_{\theta}}^{u}+e^{V_{T_{\theta}}\left(Y_{\infty}-u\right)} .
$$

Let $\zeta$ be a $\mathcal{G}_{\theta}$-measurable random variable. Since the Lévy process $V$ starts afresh at $\theta$, the conditional distribution of $Y_{\theta, \infty}$ given $(\theta, \zeta)=(t, x) \in \mathbb{Z}_{+} \times \mathbb{R}$ is the same as the distribution of $Y_{\infty}$. It follows that

$$
\mathbf{P}\left(Y_{\theta, \infty}>\zeta, \theta<\infty\right)=\mathbf{E} \bar{G}(\zeta) \mathbf{1}_{\{\theta<\infty\}}
$$

Thus, if $\mathbf{P}(\theta<\infty)>0$, then

$$
\mathbf{P}\left(Y_{\theta, \infty}>\zeta, \theta<\infty\right)=\mathbf{E}(\bar{G}(\zeta) \mid \theta<\infty) \mathbf{P}(\theta<\infty)
$$

Noting that $\Psi(u):=\mathbf{P}\left(\theta^{u}<\infty\right) \geq \mathbf{P}\left(Y_{\infty}>u\right)>0$, we deduce from this using (14) that

$$
\begin{aligned}
\bar{G}(u) & =\mathbf{P}\left(Y_{\infty}>u, \tau^{u}<\infty\right)=\mathbf{P}\left(Y_{\theta^{u}, \infty}>X_{\tau^{u}}^{u}, \tau^{u}<\infty\right) \\
& =\mathbf{E}\left(\bar{G}\left(X_{\tau^{u}}^{u}\right) \mid \tau^{u}<\infty\right) \mathbf{P}\left(\tau^{u}<\infty\right) \geq \bar{G}(0) \mathbf{P}\left(\tau^{u}<\infty\right)
\end{aligned}
$$

because $X_{\tau^{u}}^{u} \leq 0$ on $\left\{\tau^{u}<\infty\right\}$. So, we get the upper bound in (13). The lower bound is obvious.

In view of the above lemma to prove Theorem 2.3 we need to show that a finite limit $Y_{\infty}$ exists and is unbounded from above.

\section{The almost sure convergence of $Y_{n}$}

Lemma 4.1. Suppose that $\mathbf{E} e^{\varepsilon T_{1}}<\infty$ and $\mathbf{E}\left|\xi_{1}\right|^{\beta \wedge \varepsilon \wedge 1}<\infty$ for some $\varepsilon>0$ and there is $\beta>0$ such that $H(\beta)=0$. Then the sequence $Y_{n}$ tends almost surely to a finite random variable $Y_{\infty}$.

Proof. Take $p \in] 0, \beta \wedge \varepsilon \wedge 1[$. According to (12)

$$
Y_{n}-Y_{n-1}=-e^{-V_{T_{n-1}}} \int_{] T_{n-1}, T_{n}\right]} e^{-\left(V_{s-}-V_{T_{n-1}}\right)} d P_{s}=M_{1} \ldots M_{n-1} Q_{n}
$$


where

$$
M_{j}:=e^{-\left(V_{T_{j}}-V_{T_{j-1}}\right)}, \quad Q_{n}=-\int_{] T_{n-1}, T_{n}\right]} e^{-\left(V_{s-}-V_{T_{n-1}}\right)} d P_{s} .
$$

Clearly, $M_{1} \ldots M_{n-1} Q_{n}$ is the product of independent random variables, where $M_{j}$ are identically distributed and so are the random variables $Q_{n}$. Note that

$$
\mathbf{E} M_{1}^{p}=\mathbf{E} e^{-p V_{T_{1}}}=\int_{0}^{\infty} \mathbf{E} e^{-p V_{t}} F(d t)=\int_{0}^{\infty} e^{t H(p)} F(d t)<1 .
$$

Also $\mathbf{E}\left|Q_{1}\right|^{p}<\infty$ (formally we can not use Corollary 2.2 because we require here a weaker integrability condition on $\xi_{1}$ but its simple proof need only a minor change). .

Since $\mathbf{E} M_{1}^{p} \ldots M_{j-1}^{p}\left|Q_{j}\right|^{p}=\rho^{j-1} \mathbf{E}\left|Q_{1}\right|^{p}$, we have that

$$
\mathbf{E} \sum_{j \geq 1}\left|Y_{j}-Y_{j-1}\right|^{p}<\infty
$$

and, therefore, $\sum_{j \geq 1}\left|Y_{j}-Y_{j-1}\right|^{p}<\infty$ a.s.. Consequently, $\sum_{j \geq 1}\left|Y_{j}-Y_{j-1}\right|<\infty$ a.s. and, hence, the sequence $Y_{n}$ converges a.s. to the finite random variable $Y_{\infty}:=\sum_{j \geq 1}\left(Y_{j}-Y_{j-1}\right)$.

So, $Y_{\infty}$ is the sum of an absolutely convergent series. Putting $A_{0}:=1$ and $A_{n}:=M_{1} \ldots M_{n}$ we get that

$$
Y_{\infty}=\sum_{n=0}^{\infty} A_{n} Q_{n+1}=Q_{1}+M_{1} \sum_{n=1}^{\infty} \frac{A_{n}}{A_{1}} Q_{n+1}=: Q_{1}+M_{1} Y_{1, \infty}
$$

The random variable $Y_{1, \infty}$ (we abbreviate here the sum of the series starting from $n=1)$ is independent of $\left(Q_{1}, M_{1}\right)$ and has the same law as $Y_{\infty}$. Using the language of the implicit renewal theory this means that $Y_{\infty}$ is the solution of the distributional (or random) equation

$$
Y_{\infty} \stackrel{d}{=} Q+M Y_{\infty}, \quad Y_{\infty} \text { independent of }(M, Q)
$$

where $(M, Q):=\left(M_{1}, Q_{1}\right)$.

Note that

$Y_{\infty}=Q_{1}+A_{1} Q_{2}+\ldots .+A_{n-1} Q_{n}+\sum_{k=n}^{\infty} A_{k} Q_{k+1}=Q_{1}+A_{1} Q_{2}+\ldots+A_{n} Y_{n, \infty}$ 
where

$$
Y_{\infty} \stackrel{d}{=} Y_{n, \infty}:=\sum_{k=n}^{\infty} \frac{A_{k}}{A_{n}} Q_{k+1} .
$$

The following theorem adapted to our needs combines several results of the implicit renewal theory, see Th. A.6 in [6].

Theorem 4.2. Suppose that for some $\beta>0$,

$$
\mathbf{E} M^{\beta}=1, \quad \mathbf{E} M^{\beta}(\ln M)^{+}<\infty, \quad \mathbf{E}|Q|^{\beta}<\infty .
$$

Then $\lim \sup u^{\beta} \bar{G}(u)<\infty$. If the random variable $Y_{\infty}$ is unbounded from above, then $\lim \inf u^{\beta} \bar{G}(u)>0$ and in the case where the law of $\ln M$ is non-arithmetic, $\bar{G}(u) \sim C_{+} u^{-\beta}$ where $C_{+}>0$.

Proof of Theorem 2.3. We already know that the sequence of random variables $Y_{n}$ converges a.s. to a finite random variable $Y_{\infty}$ (see Lemma 4.1 whose conditions are weaker than those of Theorem 2.3). Moreover, we just proved that $Y_{\infty}$ is the solution of distributional equation (15) and $E|Q|^{\beta}<\infty$ (Corollary 2.2). If $Y_{\infty}$ is unbounded from above we can use Lemma 3.1 relating the asymptotic of the ruin probability with the tail behavior of the distribution function of $Y_{\infty}$ and get the claimed result from the theorem cited above. In the next section we show, assuming that the almost sure limit $Y_{\infty}$ exists, that $Y_{\infty}$, indeed, is always unbounded, except the case where $\sigma=0$, the random variable $\xi_{i}$ is bounded, $0<\Pi(|h|)<\infty$ and $\Pi(]-1,0[) \Pi(] 0, \infty[)=0$ for which we need the additional assumption $\mathbf{P}\left(T_{1} \leq t\right)>0$ for any $t>0$.

\section{When is $Y_{\infty}$ unbounded from above?}

We start from the following elementary consideration assuming that the limit $Y_{\infty}$ exists and is finite.

Lemma 5.1. If the random variables $Q_{1}$ and $Y_{n} / A_{n}$ for some $n \geq 1$ are unbounded from above, then $Y_{\infty}$ is unbounded from above.

Proof. Take $N$ such that $\mathbf{P}\left(Y_{\infty}>-N\right)>0$. Since $Y_{n} / A_{n}$ is unbounded from above and independent of the random variable $Y_{n, \infty}$ which has the same law as $Y_{\infty}$, we get that

$$
\begin{aligned}
\mathbf{P}\left(Y_{\infty}>0\right) & =\mathbf{P}\left(Y_{n}+A_{n} Y_{n, \infty}>0\right)=\mathbf{P}\left(Y_{n} / A_{n}+Y_{n, \infty}>0\right) \\
& \geq \mathbf{P}\left(Y_{n} / A_{n} \geq N, Y_{n, \infty}>-N\right) \\
& =\mathbf{P}\left(Y_{n} / A_{n} \geq N\right) \mathbf{P}\left(Y_{n, \infty}>-N\right)>0 .
\end{aligned}
$$


Thus, $\mathbf{P}\left(Y_{n, \infty}>0\right)=\mathbf{P}\left(Y_{\infty}>0\right)>0$. Furthermore, if the random variable $Q_{1}$ is unbounded from above and $\mathbf{P}\left(Y_{\infty}>0\right)>0$, then $Y_{\infty}$ is also unbounded from above. Indeed, take arbitrary $u>0$, then

$$
\begin{aligned}
\mathbf{P}\left(Y_{\infty}>u\right) & \geq \mathbf{P}\left(Q_{1}+A_{1} Y_{1, \infty}>u, Y_{1, \infty}>0\right) \geq \mathbf{P}\left(Q_{1}>u, Y_{1, \infty}>0\right) \\
& =\mathbf{P}\left(Q_{1}>u\right) \mathbf{P}\left(Y_{1, \infty}>0\right)=\mathbf{P}\left(Q_{1}>u\right) \mathbf{P}\left(Y_{\infty}>0\right)>0
\end{aligned}
$$

The lemma is proven.

We shall use the above lemma with $n=1$ when $Y_{1} / A_{1}=Q_{1} / A_{1}=Q_{1} / M_{1}$ or with $n=2$ when $Y_{2} / A_{2}=Q_{1} / A_{2}+Q_{2} / M_{2}$.

The arguments below use the following observation. Let $\zeta$ be a real-valued random variable and let $\eta$ be a random variable with values in a Polish space. Let $\mathbf{P}_{\zeta}$ and $\mathbf{P}_{\eta}$ be their distributions and let $\mathbf{P}_{\zeta \mid x}$ be a regular conditional distribution of $\zeta$ given $\eta=x$. If for any real $N$ the set $\left\{x: \mathbf{P}_{\zeta \mid x}([N, \infty))>0\right\}$ is not a $\mathbf{P}_{\eta}$-null set, then $\zeta$ is unbounded from above.

Lemma 5.2. Let $K>0, \sigma \neq 0$ and $t>s>0$. Then the random variables

$$
\zeta:=K e^{\sigma W_{t}}-\int_{0}^{t} e^{\sigma W_{r}} d r, \quad \tilde{\zeta}:=K e^{\sigma\left(W_{t}-W_{s}\right)}-e^{\sigma W_{t}} \int_{0}^{t} e^{\sigma W_{r}} d r
$$

are unbounded from above.

Proof. Recall that the Wiener measure charges any open ball in the space $C_{0}([0, T])$ of continuous functions $x .=\left(x_{t}\right)_{t \in[0, T]}$ with $x_{0}=0$ (in other words, it is of full support on this space). Let $g$ be a continuous function on $C_{0}([0, T])$ such that $g\left(x^{0}\right)=N$ and let $\varepsilon>0$. Then there is a $\delta>0$ such that $\left|g(x)-.g\left(x^{0}\right)\right|<\varepsilon$ on the open ball $\left\{x_{.}:\left|x_{.}-x^{0}\right|<\delta\right\}$. In particular, $g\left(x_{.}\right)>N-\varepsilon$ on this ball. With this, the claims of the lemma are obvious.

Note that in the above lemma the sign of $\sigma$ does not matter since $-W$ is again a Wiener process.

$$
\text { Let } \bar{V}:=V-\sigma W \text {. Then }
$$

$$
Q_{1}=e^{-V_{T_{1}}}\left|\xi_{1}\right|-c \int_{0}^{T_{1}} e^{-V_{r}} d r \geq\left|\xi_{1}\right| e^{-\bar{V}_{T_{1}}} e^{-\sigma W_{T_{1}}}-c \sup _{r \leq T_{1}} e^{-\bar{V}_{r}} \int_{0}^{T_{1}} e^{-\sigma W_{r}} d r .
$$

Taking into account the independence of $\xi_{1}, T_{1}, \bar{V}$ and $W$, we obtain using conditioning and Lemma 5.2, that $Q_{1}$ is unbounded from above. 
The random variable $Q_{1} / M_{1}$ is unbounded from above if and only if $\left|\xi_{1}\right|$ has this property. To cover the general case we consider the random variable

$$
\begin{aligned}
Y_{2} / A_{2} & =e^{V_{T_{2}}-V_{T_{1}}}\left|\xi_{1}\right|+\left|\xi_{2}\right|-c e^{V_{T_{2}}} \int_{0}^{T_{2}} e^{-V_{r}} d r \\
& \geq e^{V_{T_{2}}-V_{T_{1}}}\left|\xi_{1}\right|-c e^{V_{T_{2}}} \int_{0}^{T_{2}} e^{-V_{r}} d r
\end{aligned}
$$

The same arguments as above allow us to conclude that the random variable on the right-hand side is unbounded from above.

Thus, if $\sigma \neq 0$, the random variable $Y_{\infty}$ is unbounded without further assumptions.

It remains to consider the case $\sigma=0$. The further arguments are based on decompositions of Lévy processes without Gaussian component into sums of independent Lévy processes and the fact that a Poisson process has on a finite interval any number of jumps with strictly positive probability. The reasoning depends on the behavior of the Lévy measure.

1. Suppose that $\Pi(]-1,0[)>0$ and, hence, $\Pi(]-1,-\varepsilon[)>0$ for some $\varepsilon>0$. We decompose $V$ into a sum of two independent processes $V=V^{(1)}+V^{(2)}$ where $V^{(2)}=V-V^{(1)}$ and

$$
V_{s}^{(1)}:=I_{]-1,-\varepsilon[} \ln (1+x) * \mu_{s}+I_{] \varepsilon, \infty[} \ln (1+x) * \mu_{s} .
$$

Note that $V^{(1)}$ is the sum of two independent compound Poisson processes with negative and positive jumps respectively. Using conditioning with respect to the random variables $V^{(2)}, T_{1}, \xi_{1}$, which are independent of $V^{(1)}$, we easily obtain that $Q_{1}$ is unbounded from above because for any $K>0$ the random variable

$$
\zeta:=K e^{-V_{t}^{(1)}}-\int_{0}^{t} e^{-V_{r}^{(1)}} d r, \quad K>0,
$$

is unbounded from above. The latter property is clear: we may consider trajectories where $V^{(1)}$ has only negative jumps and these jumps are concentrated near $t$ leading to large values for the first term and a small impact on the value of the integral. $Y_{\infty}$

If $\left|\xi_{1}\right|$ is unbounded, then $Q_{1} / M_{1}$ is unbounded from above and, hence, so is

If $\left|\xi_{1}\right|$ is bounded and $\Pi(] 0, \infty[)>0$ (hence for some sufficiently small $\varepsilon>0$ both components of $V^{(1)}$ are nontrivial compound Poisson processes), we can 
check that $Y_{2} / A_{2}$ is unbounded from above. Indeed, from (18),

$$
Y_{2} / A_{2} \geq e^{V_{T_{2}}-V_{T_{1}}}\left(\left|\xi_{1}\right|-c e^{V_{T_{1}}} \int_{0}^{T_{1}} e^{-V_{r}} d r-c \int_{T_{1}}^{T_{2}} e^{-\left(V_{r}-V_{T_{1}}\right)} d r\right) .
$$

Using conditioning, we reduce the problem to checking that for any $t>s>0$ and $K>0$ the random variable

$$
e^{V_{t}^{(1)}-V_{s}^{(1)}}\left(K-e^{V_{s}^{(1)}} \int_{0}^{s} e^{-V_{r}^{(1)}} d r-\int_{s}^{t} e^{-\left(V_{r}^{(1)}-V_{s}^{(1)}\right)} d r\right)
$$

is unbounded from above. The compound Poisson process $\left(V_{r}^{(1)}\right)_{r \in[0, s]}$ has positive and negative jumps with intensities bounded away from zero and, therefore, for any $\varepsilon^{\prime}>0$ there is a non-null set $\Gamma_{1}$ on which

$$
e^{V_{s}^{(1)}} \leq 1, \quad \int_{0}^{s} e^{-V_{r}^{(1)}} d r \leq \varepsilon^{\prime}
$$

The process $\left(V_{r}^{(1)}-V_{s}^{(1)}\right)_{r \in[s, t]}$ is independent of $\left(V_{r}^{(1)}\right)_{r \in[0, s]}$ and has the same jump properties. For an $\varepsilon^{\prime}>0$ and $N>0$ there is a non-null set $\Gamma_{2}$ on which

$$
e^{V_{t}^{(1)}-V_{s}^{(1)}} \geq N, \quad \int_{s}^{t} e^{-\left(V_{r}^{(1)}-V_{s}^{(1)}\right)} d r \leq \varepsilon^{\prime} .
$$

Taking $\varepsilon^{\prime} \leq K / 4$ we get that on the non-null set $\Gamma_{1} \cap \Gamma_{2}$ the random variable defined in (20) is larger than $N K / 2$ and, therefore, is unbounded from above.

2. Suppose that $\Pi(] 0, \infty[)=0$ and $\Pi(|h|)=\infty$. Then for any $N>0$ there exists $\varepsilon>0$ such that $\tilde{R}_{\varepsilon}:=\Pi\left(I_{-1,-\varepsilon \mid}|h|\right) \geq N$. Let us consider the decomposition $V=V^{(1)}+V^{(2)}$ into a sum of two independent processes, this time with

$$
V_{s}^{(1)}:=I_{]-1,-\varepsilon]} h *(\mu-\nu)_{s}+I_{]-1,-\varepsilon]}(\ln (1+x)-h) * \mu_{s} .
$$

Again, even if $\left|\xi_{1}\right|$ is bounded, the random variable $Y_{2} / A_{2}$ is unbounded from above. Indeed, on the non-null set where the process $\left(V_{r}^{(1)}-V_{s}^{(1)}\right)_{r \in[s, t]}$ has no jumps and

$$
e^{V_{s}^{(1)}} \int_{0}^{s} e^{-V_{r}^{(1)}} d r \leq K / 2
$$

the random variable given by (20) dominates the value

$$
e^{\tilde{R}_{\varepsilon}(t-s)}\left(K / 2-\int_{s}^{t} e^{\tilde{R}_{\varepsilon}(s-r)} d r\right) \geq e^{N(t-s)}(K / 2-1 / N) .
$$


This implies the desired property.

The remaining subcase in which $\Pi(]-1,0[)>0$ but $\Pi(] 0, \infty[)=0$ will be considered later.

3. Suppose that $\Pi(]-1,0[)=0$ and $\Pi(h)=\infty$. Then for any $N>0$ there exists $\varepsilon>0$ such that $R_{\varepsilon}:=\Pi\left(I_{\varepsilon \varepsilon, 1]} h\right) \geq N$. We consider again a decomposition $V=V^{(1)}+V^{(2)}$ in a sum of two independent processes. The process $V^{(1)}$ is now defined as

$$
V_{s}^{(1)}:=I_{] \varepsilon, 1]} h *(\mu-\nu)_{s}+I_{] \varepsilon, 1]}(\ln (1+x)-h) * \mu_{s} .
$$

The set on which $V^{(1)}$ has no jumps on $[0, t]$ has strictly positive probability. On this set we derive the bound

$$
K e^{-V_{t}^{(1)}}-\int_{0}^{t} e^{-V_{r}^{(1)}} d r \geq K e^{R_{\varepsilon} t}-\frac{1}{R_{\varepsilon}} e^{R_{\varepsilon} t} \geq e^{N t}(K-1 / N)
$$

and we conclude as above that $Q_{1}$ is unbounded from above.

If $\left|\xi_{1}\right|$ is unbounded, then $Q_{1} / M_{1}$ is unbounded from above as well as $Y_{\infty}$.

If $\left|\xi_{1}\right|$ is bounded we again can prove that $Y_{2} / A_{2}$ is unbounded from above. Indeed, on the set on which the process $V^{(1)}$ has no jumps on $[0, s]$ (hence, it is growing linearly on this interval) we conclude that

$$
e^{V_{t}^{(1)}}\left(K e^{-V_{s}^{(1)}}-\int_{0}^{t} e^{-V_{r}^{(1)}} d r\right) \geq e^{V_{t}^{(1)}-V_{s}^{(1)}}\left(K-\frac{1}{R_{\varepsilon}}-\int_{s}^{t} e^{V_{s}^{(1)}-V_{r}^{(1)}} d r\right) .
$$

Let us define $J=I_{] \varepsilon, 1]} \ln (1+x) * \mu$, then $V_{t}^{(1)}-V_{s}^{(1)}=-R_{\varepsilon}(t-s)+J_{t}-J_{s}$ and $V_{s}^{(1)}-V_{r}^{(1)} \leq R_{\varepsilon}(t-s)-\left(J_{r}-J_{s}\right)$. Since the increment $\left(J_{r}-J_{s}\right)$ may have arbitrary many jumps (all of a size larger than $\ln (1+\varepsilon)$ ) and this happens independently of $J_{r \in[0, s]}$, we get that the random variable given by (20) is unbounded from above.

Lemma 5.3. Suppose that $\sigma=0, \Pi(]-1,0[)=0$ and $0<\Pi(h)<\infty$. If $\mathbf{P}\left(T_{1} \leq t\right)>0$ for any $t>0$, then $Y_{\infty}$ is unbounded from above.

Proof. In this case $V_{t}=-a_{h} t+L_{t}$ where the process $L_{t}:=\ln (1+x) * \mu_{t}$ is increasing. The constant $a_{h}:=\Pi(h)-a$ is strictly positive because the equality $\ln \mathbf{E} e^{-\beta V_{1}}=0$ with $\beta>0$ may hold only if $\mathbf{P}\left(V_{1}<0\right)>0$. We have

$$
Y_{n}:=-e^{-V_{-}} \cdot P_{T_{n}}=\sum_{k=1}^{n} e^{a_{h} T_{k}-L_{T_{k}}}\left|\xi_{k}\right|-c \int_{0}^{T_{n}} e^{a_{h} s-L_{s}} d s
$$


Let $\delta>0$. Put $L_{t}^{\delta}:=I_{\{x<\delta\}} \ln (1+x) * \mu_{t}$. Then for every $t \geq 0$

$$
\mathbf{E} L_{t}^{\delta}=t \Pi\left(I_{\{x<\delta\}} \ln (1+x)\right) \downarrow 0, \quad \delta \downarrow 0 .
$$

Thus, for a sufficiently small $\delta$ the set $\left\{L_{t}^{\delta} \leq 1\right\}$ is non-null. Also non-null is the set $\left\{L_{t}-L_{t}^{\delta}=0\right\}$. Their intersection, due to the independence of $L_{t}^{\delta}$ and $L_{t}-L_{t}^{\delta}$, is non-null and the larger set $\left\{L_{t} \leq 1\right\}$ is also non-null.

Take $K^{\prime}, \kappa>0$ such that $\mathbf{P}\left(1 / K^{\prime} \leq T_{1} \leq K^{\prime}\right)>0$ and $\mathbf{P}\left(\left|\xi_{1}\right|>\kappa\right)>0$. Then $\mathbf{P}\left(\inf _{k \leq n}\left|\xi_{k}\right|>\kappa\right)>0$ for each $n$ due to the independence of the random variables $\xi_{k}$.

The independence of the interarrival times and the assumption on their distribution imply that for any $n$ the set

$$
\left\{1 / K^{\prime} \leq T_{1} \leq K^{\prime}, T_{k}-T_{k-1} \leq 2^{-(k-1)}, 1<k \leq n\right\}
$$

is non-null as well as its intersection with the set $\left\{\inf _{k \leq n}\left|\xi_{k}\right|>\kappa, L_{K^{\prime}+2} \leq 1\right\}$. On this intersection, which we denote by $\Gamma_{n}$, we minorate the sum in the righthand side of (23) by replacing $L_{T_{k}}$ by unit and majorate the integral by replacing $L_{s}$ by zero. Taking into account that $T_{n} \leq T_{1}+1 \leq K^{\prime}+1$, we get in this way that

$Y_{n} \geq \frac{\kappa}{e} \sum_{k=1}^{n} e^{a_{h} T_{k}}-\frac{c}{a_{h}} e^{a_{h}\left(T_{1}+1\right)} \geq\left(\frac{\kappa}{e} n-\frac{c}{a_{h}} e^{a_{h}}\right) e^{a_{h} T_{1}} \geq\left(\frac{\kappa}{e} n-\frac{c}{a_{h}} e^{a_{h}}\right) e^{a_{h} / K^{\prime}}$

where the last inequality holds for $n \geq c e^{a_{h}+1} /\left(\kappa a_{h}\right)$. Thus, for any fixed $N$ we have on the set $\Gamma_{n}$ the bound $Y_{n} \geq N$ when $n$ is sufficiently large.

Take $y>0$ such that $\mathbf{P}\left(Y_{\infty}>-y\right)>0$. Due to the independence the set

$$
\Gamma_{n} \cap\left\{T_{n+1}-T_{n} \leq 1, Y_{n+1, \infty}>-y\right\}
$$

is non-null and on this set for sufficiently large $n$ we have that

$$
\begin{aligned}
Y_{\infty} & =Y_{n+1}+A_{n+1} Y_{n+1, \infty}=Y_{n}+A_{n} Q_{n+1}+A_{n+1} Y_{n+1, \infty} \\
& =Y_{n}+e^{a_{h} T_{n+1}-L_{T_{n+1}}}\left|\xi_{n+1}\right|-c \int_{T_{n}}^{T_{n+1}} e^{a_{h} s-L_{s}} d s+e^{a_{h} T_{n+1}-L_{T_{n+1}}} Y_{n+1, \infty} \\
& \geq Y_{n}-\left(c / a_{h}\right) e^{a_{h}\left(K^{\prime}+2\right)}-e^{a_{h}\left(K^{\prime}+2\right)} y \geq N-\left(c / a_{h}+y\right) e^{a_{h}\left(K^{\prime}+2\right)} .
\end{aligned}
$$

Since $N$ is arbitrary, the random variable $Y_{\infty}$ is unbounded from above. 
Lemma 5.4. Suppose that $\sigma=0, \Pi(] 0, \infty[)=0$, and $0<\Pi(|h|)<\infty$. If $\mathbf{P}\left(T_{1} \leq t\right)>0$ for any $t>0$, then $Y_{\infty}$ is unbounded from above.

Proof. In this case $V_{t}=b_{h} t-L_{t}$ where the process $L_{t}:=-\ln (1+x) * \mu_{t}$ is increasing and the $b_{h}:=a-\Pi(h)>0$. We have

$$
Y_{n}:=-e^{-V_{-}} \cdot P_{T_{n}}=-\sum_{k=1}^{n} e^{-b_{h} T_{k}+L_{T_{k}}} \xi_{k}-c \int_{0}^{T_{n}} e^{-b_{h} s+L_{s}} d s .
$$

As in the proof of the previous lemma, we get that for any $t \geq 0$ the set $\left\{L_{t} \leq 1\right\}$ is a non-null set, there are constants $\kappa$ and $K^{\prime}$ such that $\mathbf{P}\left(\left|\xi_{k}\right|>\kappa\right)>0$ for each $k$ and $\mathbf{P}\left(1 / K^{\prime} \leq T_{1} \leq K^{\prime}\right)>0$. For any $n$ the set

$$
\left\{1 / K^{\prime} \leq T_{1} \leq K^{\prime}, T_{k}-T_{k-1} \leq 2^{-(k-1)}, 1<k \leq n\right\}
$$

is non-null and its intersection with the set $\left\{\inf _{k \leq n}\left|\xi_{k}\right|>\kappa, L_{K^{\prime}+2} \leq 1\right\}$ is nonnull. On this intersection, denoted $\Gamma_{n}$,

$$
Y_{n} \geq \kappa e^{-b_{h} T_{1}} \sum_{k=1}^{n} e^{-b_{h}\left(T_{k}-T_{1}\right)}-\frac{c e}{b_{h}} \geq \kappa e^{-b_{h}\left(K^{\prime}+1\right)} n-\frac{c e}{b_{h}} \geq N
$$

for any $N$ when $n$ is sufficiently large.

Take $y>0$ such that $\mathbf{P}\left(Y_{\infty}>-y\right)>0$. Then the set

$$
\Gamma_{n} \cap\left\{T_{n+1}-T_{n} \leq 1, Y_{n+1, \infty}>-y\right\}
$$

is non-null and on this set for sufficiently large $n$ we have that

$$
\begin{aligned}
Y_{\infty} & =Y_{n}+e^{-b_{h} T_{n+1}+L_{T_{n+1}}}\left|\xi_{n+1}\right|-c \int_{T_{n}}^{T_{n+1}} e^{-b_{h} s+L_{s}} d s+e^{-b_{h} T_{n+1}+L_{T_{n+1}}} Y_{n+1, \infty} \\
& >Y_{n}-c e-e y \geq N-e(c+y) .
\end{aligned}
$$

Since $N$ is arbitrary, the random variable $Y_{\infty}$ is unbounded from above.

\section{References}

[1] Albrecher H., Constantinescu C., Thomann E., Asymptotic results for renewal risk models with risky investments. Stoch. Proc. Appl. 122 (11) (2012), 3767-3789. 
[2] Eberlein E., Kallsen J., Mathematical Finance. Springer, Cham (2019).

[3] Frolova A., Kabanov Yu., Pergamenshchikov S., In the insurance business risky investments are dangerous. Finance and Stochastics 6 (2) (2002), 227-235.

[4] Grandell I., Aspects of Risk Theory. Springer, Berlin (1990).

[5] Jacod J., Shiryaev A.N., Limit Theorems for Stochastic Processes. 2nd edition, Springer, Berlin (2002).

[6] Kabanov Yu., Pergamenshchikov S., Ruin probabilities for a Lévy-driven generalised Ornstein-Uhlenbeck process. Finance and Stochastics 24 (1) (2020), 39-69.

[7] Kabanov Yu., Puchlyakov N., Ruin probabilities with investments: smoothness, IDE and ODE, asymptotic behavior. Preprint (2020).

[8] Paulsen J., Risk theory in stochastic economic environment. Stoch. Proc. Appl. 46 (2) (1993), 327-361.

[9] Paulsen J., Stochastic Calculus with Applications to Risk Theory. Lecture Notes, Univ. of Bergen and Univ. of Copenhagen (1996).

[10] Paulsen J., Sharp conditions for certain ruin in a risk process with stochastic return on investments. Stoch. Proc. Appl. 75 (1) (1998), 135-148.

[11] Paulsen J., Gjessing H.K., Ruin theory with stochastic return on investments. Adv. Appl. Probab. 29 (4) (1997), 965-985. 